\title{
Reverse diastolic flow in the common carotid artery in severe aortic regurgitation, causing brain ischemia
}

\author{
Naoto Morimoto, MD, Keisuke Morimoto, MD, Yoshihisa Morimoto, MD, Toshihito Sakamoto, MD, \\ Masamichi Matsumori, MD, Kenji Okada, MD, and Yutaka Okita, MD, Kobe, Japan
}

$\mathrm{D}$ iastolic reverse flow in the common carotid artery (CCA) at duplex sonography can be seen in patients with severe aortic regurgitation (AR). We report a patient with severe AR whose diastolic reverse flow in the right CCA caused transient brain ischemia. To our knowledge, there are no descriptions of diastolic steal volume by AR causing cerebral ischemia. This is the first description of this rare pathophysiologic form of cerebral ischemia.

\section{Clinical Summary}

A 70-year-old woman had undergone partial arch replacement and coronary bypass grafting for acute aortic dissection complicated by a large myocardial infarction resulting from the malperfused left coronary system. Although postoperative echocardiography showed mild aortic and mitral regurgitation, AR and mitral regurgitations gradually worsened during the next 8 months, which resulted in repeated hospitalizations for congestive heart failure. The patient had temporary blurred vision in her right eye. This symptom was accompanied by dizziness, headache, motor weakness of the right forearm, and pain migrating from the right shoulder to the right forearm. These symptoms began on sitting up in bed and spontaneously resolved within 10 minutes. Duplex sonography revealed diastolic reverse flow in the right CCA with a peak systolic velocity of 58 $\mathrm{cm} / \mathrm{s}$ and an end-diastolic velocity of $-54 \mathrm{~cm} / \mathrm{s}$ (Figure 1, $C$ ). The velocity time integral of the antegrade flow was $16.7 \mathrm{~cm}$, the velocity time integral of the retrograde flow was $10.2 \mathrm{~cm}$, and the regurgitation rate was $61.1 \%$. Acetazolamide-activated brain perfusion ${ }^{123}$ I-IMP single photon emission computed tomography showed severe hypoperfusion to the right middle cerebral artery area. In $43.7 \%$ of this region, cerebral blood flow at rest was decreased to less than $15 \mathrm{~mL} / 100 \mathrm{~g} / \mathrm{min}$ on stereotactic extraction estimation (Figure 2, A). Magnetic resonance angiography of the neck and head demonstrated the absence of the right anterior and posterior communicating arteries as collateral circulation.

From the Division of Cardiovascular Surgery, University of Kobe, Kobe, Japan.

Received for publication Dec 19, 2007; accepted for publication Jan 15, 2008.

Address for reprints: Naoto Morimoto, MD, 7-5-2, Kusunoki-cho, Chuo-ku, Kobe, Hyogo, Japan, 650-13 (E-mail: naotom@med.kobe-u.ac.jp).

J Thorac Cardiovasc Surg 2008;135:1176-7

$0022-5223 / \$ 34.00$

Copyright $\odot 2008$ by The American Association for Thoracic Surgery doi:10.1016/j.jtcvs.2008.01.010
Surgery was performed by a right thoracotomy via the fourth intercostal space. Cardiopulmonary bypass was established using ascending aorta and bicaval cannulation with the aid of vacuumassisted venous drainage. Cardioplegic arrest was achieved by using antegrade and retrograde cold blood cardioplegia. The aortic and mitral valves were replaced by a Carpentier-Edwards (Edwards Lifesciences, Irvine, Calif) bioprosthesis.

The postoperative course was uneventful. The carotid scan demonstrated normal positive diastolic flow in the right CCA (Figure 1, $D$ ). The postoperative brain single photon emission computed tomography scan showed the normalization of the cerebral perfusion (Figure 2, B). The patient currently has no neurologic symptoms with New York Heart Association class II.

\section{Discussion}

Diastolic reverse flow in the carotid arteries that persists more than $50 \%$ of the duration of diastole is an abnormal waveform on carotid Duplex scanning, although identification of a small postsystolic reversal is a normal finding. The mechanism of reverse diastolic flow is the rapid ejection of the increased ventricular volume that exists in $\mathrm{AR}$, producing a suction or Venturi effect within the aorta and its branches. ${ }^{1}$ Another possible mechanism is that increased distensibility of the aorta, being replaced with a vascular graft, can cause increased systolic expansion and diastolic recoil of the vessel. ${ }^{2}$ Malaterre and colleagues ${ }^{3}$ reported reverse flow only in patients with grade III or IV AR. Kallman ${ }^{4}$ reported the same abnormal carotid flow pattern in 3 patients with severe AR who were scheduled for aortic valve replacement. All had normal carotid patterns postoperatively. In the present case, diastolic reverse flow was normalized after aortic valve replacement.

In the present case, duplex sonography, magnetic resonance angiography, and single photon emission computed tomography findings indicated the 3 following facts in addition to diastolic reverse flow. (1) Reverse diastolic flow reduced total antegrade flow in the right carotid arteries. (2) The right middle cerebral artery received its blood flow from only the right internal carotid artery without any collateral supply. (3) The right middle cerebral artery territory was subject to ischemia with its depressed flow reserve. Reverse diastolic flow decreases total antegrade flow as a natural consequence. Reduced total antegrade flow in the right carotid system and an anatomic abnormality of the circle of Willis were attributed to cerebral hypoperfusion in this patient. Moreover, in patients with severe AR the collapsing pulse and maximal reduction of the mean arterial blood pressure cause the reduction of the autoregulatory capacity of cerebral blood flow, which might decrease the tolerance for brain ischemia. These mechanisms are compatible with the fact that brain ischemia was resolved after the disappearance of AR. 


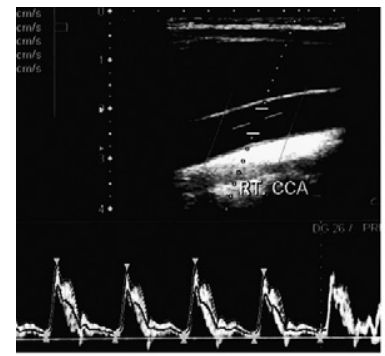

A

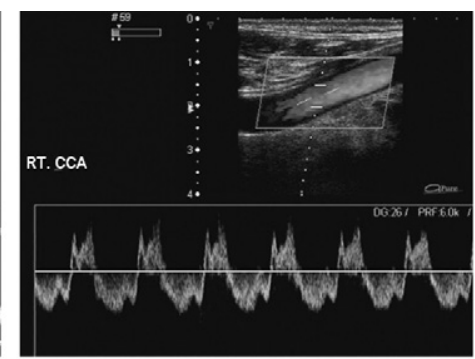

B

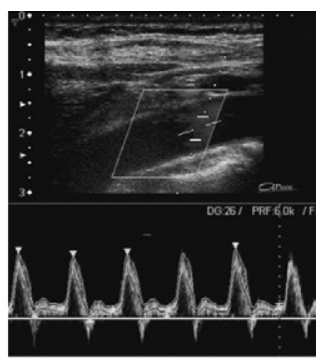

C

Figure 1. Serial duplex scans of right CCA. Although carotid flow pattern was normal after previous aortic surgery $(A)$, diastolic flow was reversed before aortic valve surgery (B). After aortic valve replacement, diastolic flow pattern was normalized (C). $R T$, : $C C A$, right common carotid artery.
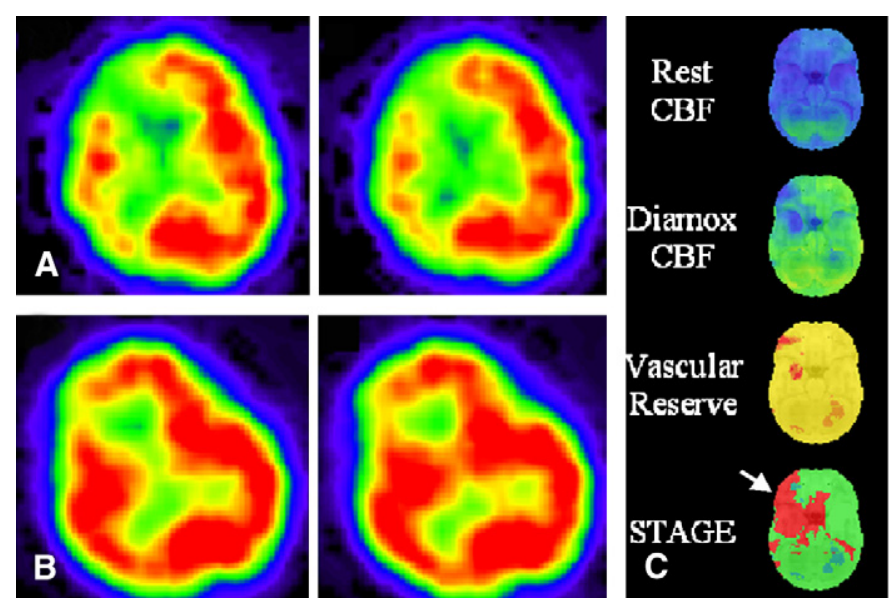

Figure 2. A, Rest images of ${ }^{123}$-IMP single photon emission computed tomography. Before aortic valve replacement, cerebral blood flow was impaired in most of the right middle cerebral artery territory. B, After the procedure, whole brain circulation was normalized. Images of stereotactic extraction estimation before (C) and after (D) aortic valve replacement. Staging map images show that resting cerebral blood flow was reduced in the anterior half of the right middle cerebral artery territory before the surgery and was normalized after surgery. $C B F$, Cerebral blood flow.

\section{Conclusions}

If a patient with severe AR has any neurologic symptoms, a carotid duplex scan should be performed because of the possibility of brain ischemia caused by diastolic reverse flow.

\section{References}

1. Fleming ME. The mechanism of the pulsus bisferiens. Br Heart J. 1957; 19:519-24.
2. Mori K, Hayabuchi Y, Kuroda Y, Nii Y, Yuasa Y, Taguchi Y. Retrograde holodiastolic flow in the abdominal aorta detected by pulsed Doppler echocardiography in patients with Kawasaki disease. Eur J Pediatr. 2000;159:509-14.

3. Malaterre HR, Kallee K, Giusiano B, Letellec L, Djiane P. Holodiastolic reversal flow in the common carotid: another indicator of the severity of aortic regurgitation. Int J Cardiovasc Imag. 2001;17:333-7.

4. Kallman CE, Gosink BB, Gardner DJ. Carotid duplex sonography bisferious pulse contour in patients with wortic valvular disease. Am J Roentenol. 1991;157:403-7. 